\title{
Ventajas y desventajas en la enseñanza de la composición musical en medio de una pandemia
}

\section{Advantages and disadvantages of teaching music composition in the midst of a pandemic}

\author{
Diego Graciosi ${ }^{1}$ \\ https://orcid.org/0000-0002-3056-6940 \\ diegograciosi@yahoo.com.ar \\ Facultad de Arte | Universidad Nacional \\ de La Plata | Argentina
}

\author{
Pedro Hernández ${ }^{2}$ \\ https://orcid.org/0000-0001-6962-6974 \\ esteledhel@yahoo.com.ar \\ Facultad de Arte | Universidad Nacional \\ de La Plata | Argentina
}

\section{RESUMEN}

¿La enseñanza de la composición musical en la virtualidad puede desarrollarse?... ¿Cómo?

Solemos decir entre docentes y muchas veces a los/as alumnos/as que no se enseña a componer música, lo cual genera una crisis inicial a quienes llegan a la Facultad de Artes (FDA) en la Universidad de La Plata (UNLP) para aprender a componer música. Hay una metáfora que utilizamos para describir el paso por esta carrera universitaria que es la de la valija de herramientas. Quienes se inscriben en la materia llegan a la FDA con diferentes realidades, algunos/as tienen más herramientas que otros en sus valijas, hay quienes las saben usar más, hay quienes menos...otros/as inclusive no poseen ninguna, y en el transcurso de cada clase, de cada intercambio grupal e individual, se va construyendo una vinculación que nos permite compartir experiencias concretas de este oficio.

\section{ABSTRACT}

Can the teaching of musical composition in virtuality be developed? ... How?

We tend to say among teachers and many times to students that they are not taught to compose music, which generates an initial crisis for those who come to the Faculty of Arts (FDA) at the University of La Plata (UNLP) to learn to compose music. There is a metaphor that we use to describe the passage through this university career, which is that of the tool bag. Those who enroll in the matter come to the FDA with different realities, some have more tools than others in their suitcases, there are those who know how to use them more, there are those who less ... others do not even have any, and in the course of each class, each group and individual exchange, a bond is built that allows us to share concrete experiences of this trade.

\footnotetext{
${ }^{1}$ Titular de la asignatura Taller de composición musical, Departamento de música, FDA, UNLP.

2 Jefe de trabajos prácticos de la asignatura Taller de composición musical, Departamento de música, FDA, UNLP.
}

PALABRAS CLAVE Composición, Música,

Taller, Educación Virtual.

\section{KEY WORDS}

Composition,

Music,

Workshop,

Virtual Education. 


\section{PRESENTACIÓN}

Como docentes esta pandemia nos ha puesto en un escenario en el cual nos hemos encontrado con una valija en donde había pocas herramientas para desarrollar la labor que estamos acostumbrados a desarrollar de una determinada manera.

Esto que nos sucede es algo que a los/as alumnos/as de composición musical solemos decirles con regularidad: "debemos ser creativos tanto con las herramientas que tenemos como con los instrumentos y músicos que están a nuestro alrededor. No solo debemos ser creativos en el desarrollo de la escritura de una música en particular, sino que debemos ser creativos para organizar conciertos, estimular el potencial de otros músicos, generar nuevos vínculos, etc., tenemos que ser productores además de compositores", esto, claro no implica un pensamiento derrotista ni conformista, sino por lo contrario, busca despertar en el alumnado el deseo de crear concientemente y en contacto con la realidad.

Hoy los docentes, en particular los que ocupamos cargos en composición musical, debemos desarrollar la creatividad para llevar adelante la transmisión de los contenidos de una asignatura que consiste en hacer música en un sentido bastante comprensivo, esto quiere decir: escribir música, tocar música, hacer música en grupo o individualmente. En muchos casos hemos podido sostener algunas formas de enseñar y transmitir contenidos, y en muchas más de las que queríamos no. Eso nos obligó a valernos de nuevas herramientas que estaban a disposición y por comodidad y miedo no las usábamos. Es el caso de AulasWeb.

Este paso, obligado, nos permitió patear el tablero y redescubrir contenidos ocultos u olvidados de la asignatura que desembocaron en salidas positivas para acercarnos a esta transmisión reflexiva de la composición musical. Las nuevas líneas pedagógicas en asignaturas como matemáticas hablan de validar los diferentes caminos que se toman para llegar al mismo resultado, hemos y seguimos trabajando 
para descubrir nuevas estrategias de transmisión de experiencias y conocimientos que aporten al despertar de la curiosidad. Porque la creatividad requiere de curiosidad, y en tiempos de pandemia hemos visto que las innumerables variables de deserción terminan agrietando los estados de creatividad. Por lo tanto el trabajo de estimulación para la creación ha sido un desafío mayor. Mariano Etkin, quien fue docente titular de las cátedra de composición musical de FDA, UNLP a mediados de la década del 90' dijo que "a un alumno de composición no se le puede enseñar a desear", y coincido, pero sí se puede despertar el deseo. Es aquí donde veo el lado positivo de esta situación sin precedentes que estamos atravesando a nivel mundial, hemos estado ante una realidad que nos ha dado poco margen para comodidad, hemos estado incómodos, y en esa incomodidad estamos aprendiendo a ser creativos sabiendo que la virtualidad nos priva de muchas herramientas irremplazables, pero aporta muchas otras que hoy en día ayudan a llevar adelante una cursada y en el futuro posiblemente sea un gran aporte en la presencialidad.

\section{Este paso, obligado, nos permitió patear el tablero y redes- cubrir contenidos ocultos u olvidados de la asignatura que desembocaron en salidas positivas para acercarnos a esta transmisión reflexiva de la composición musical.}

En realidad, el hilo conductor de la enseñanza no se pierde, la crisis está, si podemos llamarla de esta manera, en ser creativos a corto plazo.

\section{REALIDAD QUE MODIFICA LO ESTABLECIDO}

En nuestro caso como equipo de cátedra junto al cuerpo docente que integramos la cátedra Taller de Composición Musical asignatura que pertenece al Ciclo de Formación Musical Básico (CIFOMUBA), del departamento de música de la Facultad de Artes de la Universidad Nacional de La Plata, este ciclo lectivo presente nos ha puesto en la situación de necesitar aplicar en nuestra metodología de enseñanza lo que solemos promover con los alumnos en sus creaciones musicales... adaptarnos a las posibilidades que tenemos frente a nosotros y con ello crear algo nuevo, o como bien se dice hoy en día... algo innovador. Es decir, algo que ya está pero que las múltiples y diversas miradas hacen que se resignifique y que podamos adoptar de manera renovada en el aquí y ahora. Por lo tanto, siempre hay una nueva manera de enseñar. Además, debemos tener en cuenta que, así como las generaciones se van renovando también debemos renovarnos no- 
sotros, en relación a los contenidos y también la forma de abordarlos.

Cambian los paradigmas para la conexión con los alumnos y nos cambian las bases desde donde enseñamos.

Claro que este cambio de paradigma ha implicado también tener un mayor grado de atención a casos particulares de alumnos/as que requerían una contención mayor para desarrollarse en su primer año en la Facultad de Artes y en la mayoría de los casos, al ser ingresantes, su primer año en la universidad. Desde el inicio de esta asignatura en el año 2006 hemos trabajado con muchas satisfacciones en el proceso de cada trabajo práctico así como también en el punto de cierre de cada unidad temática, siempre tomando como herramienta pedagógica la metodología de taller. Esto nos permite desarrollar tres puntos relacionados con la integración desde la práctica musical:

1. Integrar a todo el alumnado ingresante con su heterogeneidad.

2. Utilizar la práctica concreta de la música en el aula como medio de debate, intercambio colectivo y retroalimentación constante.

3. Hacer música para luego hablar desde una mirada más analítica y teórica.

Estos tres aspectos se vieron claramente modificados pero no neutralizados por completo.

El primer aspecto, en lo que refiere a la integración en donde específicamente no aludimos a los contenidos previos solamente, hemos notado también que la desigualdad de condiciones socioeconómicas ha repercutido un paso antes que el conocimiento musical previamente adquirido de manera más notoria que en ciclos lectivos anteriores. Por lo tanto la capacidad de adaptación atravesada sobre la que estamos reflexionando en el presente escrito tuvo uno de sus primero puntos de prueba. Hemos tenido que brindar un abanico de posibilidades para lograr tener intercambio, conexión, diálogo, etc. de manera virtual, que se encuentre al alcance de los/as alumnos/as.

En nuestro caso como cátedra teníamos y tenemos a nuestro alcance determinados recursos desde un aspecto material, pero no de uso técnico cotidiano. Algunos docentes poseen un mayor bagaje del manejo tecnológico.

El segundo punto que es uno de los más ricos de la metodología de taller es el hacer en el aula de manera colectiva y el intercambio general que esto produce. Si bien las plataformas, como es el caso de Zoom, permiten escuchar y ver música entre docentes y alumnos/as, la frialdad de la distancia física impactó en un principio de manera negativa la fluidez de las interacciones. La ausencia de alumnos con cámaras para su conexión en clase, en otros casos la deficiencia de la conexión de internet, en otros la falta de micrófonos son algunos de los factores que dificultan enormemente la fluidez de un intercambio o debate 
sobre la música en la cual estamos trabajando. No perdemos de vista que al ser ingresantes de la carrera apenas han podido intercambiar de manera presencial al comienzo de este año (2020) en la Facultad solamente durante 3 semanas el curso de ingreso, sin contar con ninguna clase presencial de cada materia debido al comienzo de la ASPO un día antes de las clases inaugurales. Por ello hemos desarrollado maneras y espacios virtuales de intercambio como lo son los trabajos grupales (a distancia) tanto en horarios fuera de la cursada virtual, como dentro de una misma cursada realizando la división de salas en la plataforma Zoom, teniendo resultado muy buenos a tal punto que ha surgido de parte de los alumnos, como una autogestión, la idea de crear un espacio en la plataforma Soundcloud en donde han expuesto todos sus trabajos de composición musical.

\section{https://soundcloud.com/tallerdecompo2020}

Si bien las plataformas, como es el caso de Zoom, permiten escuchar y ver música entre docentes y alumnos/as, la frialdad de la distancia física impactó en un principio de manera negativa la fluidez de las interacciones. La ausencia de alumnos con cámaras para su conexión en clase, en otros casos la deficiencia de la conexión de internet, en otros la falta de micrófonos son algunos de los factores que dificultan enormemente la fluidez de un intercambio o debate sobre la música en la cual estamos trabajando.

Este espacio virtual en donde los/as alumnos/as comparten su música ya no como trabajos prácticos, sino como creaciones musicales en sí mismas nos ha dado la pauta del significado del espacio curricular en estos momentos que estamos transitando.

El tercer aspecto es el que tuvo un mayor desarrollo debido a la creación de un nuevo espacio virtual de la asignatura en AulasWeb y la creación de material audiovisual complementario para lograr un mayor acompañamiento de los contenidos de cada unidad temática.

Hemos generado nuevos materiales de cátedra para el apoyo del seguimiento de cada unidad y sus contenidos:

1. Nuevo material audiovisual que antes solo existía como recomendación de una bibliografía complementaria.

2. Nuevas clases profundizando y reflexionando contenidos relacionados con temáticas específicas de la composición musical.

3. Organización de todo el material bibliográfico obligatorio y de consulta de manera más clara y directa para su acceso de manera virtual, gracias a AulasWeb. 
El aula presencial en realidad no tiene reemplazo. Lo que existen son alternativas o planes $B$, y hasta $C$. Son planes útiles, sostienen un hilo conductor de la currícula pero la vinculación social se pierde.

Como hemos expresado anteriormente la vinculación con los alumnos ha sido por diferentes vías de comunicación: E.mail, AulasWeb, Zoom, Facebook, Blog. En un principio todos fueron recursos pertinentes para cerciorarnos de que el/la alumno/a estuviese conectado, y sobre todo vinculado, con la asignatura.

Toda asignatura termina de completarse con el alumnado, no solo es un conjunto de docentes. Esta situación mundial sin precedentes nos ha puesto en un estado mayor de sensibilidad con la necesidad de fortalecer el vínculo docente-alumno/a, y reforzar la conciencia de que el conocimiento se desarrolla en dicha vinculación volviéndose multidireccional el aprendizaje.

Específicamente para dar respuesta a la situación crítica en la que nos hallamos (ya que hace 15 años manejamos la mecánica de clase presencial con esta asignatura) tuvimos que sumergirnos en el manejo lo virtual de manera inmediata, sin tener un conocimiento profundo, ya que la capacitación recibida anteriormente era poca y la necesidad nos llevó a contactarnos con otros/as docentes, para recibir consejos y despejar dudas. Gracias a esos vínculos logramos aprovechar con mayor eficiencia los elementos y posibilidades de AulasWeb como herramienta prioritaria para la vinculación con los alumnos en reemplazo del aula física, aunque sabemos que este es también un aprendizaje que nos llevará más tiempo a irá puliendo nuestras habilidades en este medio.

\section{Específicamente para dar respuesta a la situación crítica en la que nos hallamos (ya que hace 15 años manejamos la mecáni- ca de clase presencial con esta asignatura) tuvimos que sumer- girnos en el manejo lo virtual de manera inmediata, sin tener un conocimiento profundo, ya que la capacitación recibida an- teriormente era poca y la necesidad nos llevó a contactarnos con otros/as docentes, para recibir consejos y despejar dudas.}

El 2020 como año lectivo trajo, junto con nuevos problemas, nuevas perspectivas: de acercamiento y revisión a los contenidos y prácticas. La habituación a un sistema nos ha hecho, muchas veces, pasar por alto algunas inconsistencias o elementos dejados de lado en el transcurso de la currícula, confiados en nuestra mecánica y seguros en nuestra metodología (en base a la exitosa experiencia acumulada en 15 años de trabajo). La nueva situación nos obligó a repensar nuestra 
planificación, y actuar en consecuencia, renovando materiales, ampliando contenidos, diversificando los medios de vinculación y revisando críticamente nuestras prácticas previas. Todo esto ha resultado en una profunda renovación tanto de nuestra materia globalmente como de nuestra búsqueda personal y profesional, conectándonos con ideas y proyectos que estaban archivados dentro de la implacable rutina organizada que habíamos logrado construir. La crisis nos posicionó frente a nosotros mismos como docentes e investigadores en un escenario frágil que nos exigió introspección para lograr un cambio deseado pero postergado.

La crisis nos posicionó frente a nosotros mismos como docentes e investigadores en un escenario frágil que nos exigió introspección para lograr un cambio deseado pero postergado.

Como complemento para este informe hemos realizado una estadística de la descensión de alumnos en el año presente y otra promedio de la matrícula de los últimos tres años (2017/18/19) para ver cómo en un número inicial de alumnos inscriptos en la asignatura los niveles de deserción han sido mayores en este año.

Asignatura: Taller de composición musical. Departamento de Música. FDA - UNLP.

Matrícula de ingreso 2020

\begin{tabular}{|c|c|c|c|c|c|c|c|c|c|}
\hline Mes & marzo & abril & mayo & junio & julio & agosto & $\begin{array}{c}\text { septiem- } \\
\text { bre }\end{array}$ & octubre & noviembre \\
\hline $\begin{array}{c}\text { Cantidad } \\
\text { de alumnos }\end{array}$ & 100 & 60 & 56 & 50 & 50 & 47 & 36 & 29 & 23 \\
\hline
\end{tabular}

Matrícula de los últimos 3 años (2017/18/19)

El número de alumnos/as ingresantes entre estos tres años oscila entre 115 y 97 alumnos.

\begin{tabular}{|c|c|c|c|c|c|c|c|c|c|}
\hline Mes & marzo & abril & mayo & junio & julio & agosto & $\begin{array}{c}\text { septiem- } \\
\text { bre }\end{array}$ & octubre & noviembre \\
\hline $\begin{array}{c}\text { Cantidad } \\
\text { de alumnos }\end{array}$ & 100 & 75 & 70 & 60 & 60 & 55 & 50 & 40 & 40 \\
\hline
\end{tabular}


Existen momentos que son irremplazables como lo son la relación entre el intérprete o instrumentista y el compositor, es una instancia de la creación en la cual a través de esta vinculación social se alcanza un momento de relación con el sonido inigualable. Estos momentos no los hemos podido generar, pero si nos hemos encontrado de manera virtual, que claro no es lo mismo que la presencialidad, que nos ha permitido escuchar, comprender, crear y comenzar.

En momentos de extrema dificultad, como este, se dan saltos de crecimiento, e indudablemente este ha alimentado el proyecto educativo del que somos generadores y parte activa, obligándonos a observarnos crítica y constructivamente, para avanzar e incorporar los elementos necesarios para concretar ese crecimiento.

Hoy seguimos aprendiendo, incluyendo, trascendiendo limitaciones, abriendo nuevas perspectivas para el desarrollo de nuestro espacio de enseñanza, incluyendo al cuerpo docente, alumnos/as, directivos... es el comienzo de una nueva etapa de inclusividad. Pese a las innumerables crisis de adaptación que hemos tenido que atravesar todos/as rescatamos como cátedra el valor de nuevo. 


\section{BIBLIOGRAFÍA}

Fisherman, Diego (2011). "Después de la música. El siglo XX y más allá." Ed. Eterna Cadencia, 2011.

Gandini, Gerardo (1998). “Del recato y otros pudores. Reflexiones sobre el oficio del componer". Ponencia -Tercera Reunión de Arte Contemporáneo, Santa Fe, Argentina, octubre 1997. Publicada en sus anales y en Pauta №65, México D.F., Enero-Marzo 1998.

Nono, Luigui. "El error como necesidad" Texto que salió originalmente en una edición especial de la revista francesa de música contemporánea “Contrechamps". Traducido por Gabriel Paiuk. Disponible en: https://consonanzastravaganti.blogspot.com/2011/06/luigi-nono-el-error-como-necesidad.html?view=classic

Ramón López Martín "Reflexiones Educativas para el pos Covid-19. Recordando el Futuro" Revista Internacional de Educación para la Justicia Social, 2020, 9(3e), 127-140.

\section{OTRAS CONSULTAS}

"ARTESANO DE FIN DE SIGLO. Maestros en el arte". Collage cultural originalmente realizado en 1995 por Claudio Koremblit: Disponible en:

https://www.youtube.com/watch?v=_FdU3TZdolM 